\title{
Neonatal Marfan syndrome diagnosed prenatally
}

\author{
Anna Wójtowicz , Dagna Ochrem ${ }^{1}$, Artur Dobosz ${ }^{2}$, Hubert Huras ${ }^{1}$, \\ Maria Respondek-Liberska ${ }^{3}$ \\ ${ }^{1}$ Department of Obstetrics and Perinatology, Jagiellonian University Medical College, Krakow, Poland \\ ${ }^{2}$ Department of Medical Genetics, Jagiellonian University Medical College, Krakow, Poland \\ ${ }^{3}$ Department of Prenatal Cardiology, Polish Mother's Memorial Hospital-Research Institute, Lodz, Poland
}

Neonatal Marfan syndrome (nMFS) is far less common than the classic type and is considered to be a separate clinical entity, with its unique symptoms, specific mutations in the FBN1 gene, and extremely poor prognosis. So far, all reported cases have been sporadic and resulted from de novo mutations in the region encompassing exons 24-32 of the FBN1 gene. The lifespan of the affected newborns is greatly diminished due to rapidly progressive cardiovascular disease, entailing death within the first year of life for the majority of the cases. We present a patient with nMFS which was suspected prenatally based on serial ultrasound and targeted echocardiography examinations. To the best of our knowledge, this has been the tenth case of MFS diagnosed prenatally reported so far in the literature, and the fifth with the diagnosis being confirmed by molecular testing.

A 34-year-old secundipara (history of 1 uncomplicated pregnancy, spontaneous term delivery, healthy newborn, normal development) was referred at 24 weeks to our Department for detailed ultrasound due to protosystolic tricuspid regurgitation, choroid plexus bilateral cysts, and right kidney pyelectasis. First-trimester ultrasound screening showed no abnormalities and the risk of aneuploidy was calculated as low. nMFS was suspected based on cardiomegaly, regurgitation of the mitral, tricuspid and aortic valve (Figure 1), enlargement of the ascending aorta (Figure 2), dilatation of sinus Valsalva of the aorta with communication with the left ventricle, as well as humerus and femur length exceeding 2SD for gestational age, abnormal fetal profile and hand - with long fingers and contractures of the joints. The fetus (sex male) had detailed echocardiography monitoring in the second half of pregnancy due to progression of cardiomegaly, and echocardiographic features of congestive heart failure (Figure 3). Despite transplacental treatment with digoxin, fetal demise occurred at 35 weeks of gestation. The post mortem inspection revealed the following: down-slanting palpebral fissures, crumpled ears, micrognathia, hand joint contractures (Figure 4), senile look, tall stature, arachnodactyly (hand middle finger of $4 \mathrm{~cm}$ with mean value for gestational age of $2.6-2.7 \mathrm{~cm}$ ), elongated extremities, as well as prenatally described cardiac anomalies. A skin fragment sampled during autopsy was used for fibroblasts culture. DNA isolated from skin fibroblasts was consecutively subjected to array CGH and sequence analysis of the FBN1 gene. Array CGH with the cut-off value of 200.000 bp did not detect any deletions or duplications. On the contrary, sequencing of genomic DNA comprising coding region of the FBN1 gene with neighboring intronic sequences using Sanger method on ABI3500 device revealed the presence of $\mathrm{c} .3964+1 \mathrm{G}>\mathrm{C}$ heterozygous splice site mutation preceding exon 32 , which predisposes to skipping exons during protein production, thus making the protein deficient. The same mutation was previously described in one patient with nMFS. Therefore, it is believed to be associated with severe phenotype of the affected individuals.

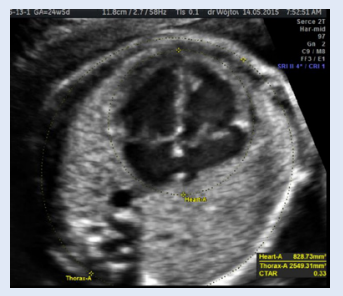

Figure 1. Four chamber view at 24 weeks

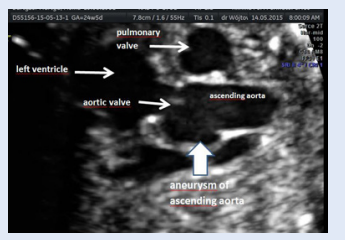

Figure 2. Left ventricle outflow tract at 24 weeks

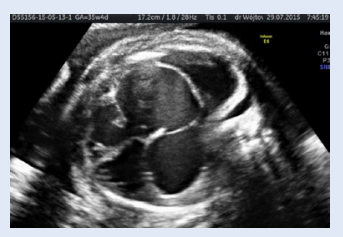

Figure 3. Four chamber view at 35 weeks

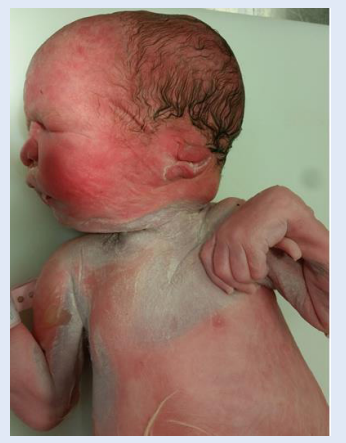

Figure 4. Neonatal Marfan syndrome features in the face and hand 